\title{
Natural convection in eccentric spherical annuli
}

\author{
A. D. Gallegos ${ }^{\dagger} \quad$ C. Málaga ${ }^{\dagger *}$
}

\begin{abstract}
A fluid between two spheres, concentric or not, at different temperatures will flow in the presence of a constant gravitational force. Although there is no possible hydrostatic state, energy transport is dominated by diffusion if temperature difference between the spheres is small enough. In this conductive regime the average Nusselt number remains approximately constant for all Rayleigh numbers below some critical value. Above the critical Rayleigh number, plumes appear and thermal convection takes place. We study this phenomenon, in particular the case where the inner sphere is displaced from the centre, using a two-component thermal lattice Boltzmann method to characterize the convective instability, the evolution of the flow patterns and the dependence of the Nusselt number on the Rayleigh number beyond the transition.
\end{abstract}

\section{Introduction}

Natural convective flow between a sphere at constant temperature and its spherical enclosure at a different temperature is an idealisation of many problems of practical interest. Convection patterns between concentric spherical shells were first observed by Bishop et al. in 1966 for a range of aspect ratios [1. Experiments were performed with the inner sphere hotter than the outer sphere. A wide variety of steady and non steady patterns were observed in subsequent experiments using air, water and silicone oil; including the case of vertically eccentric spherical shells 2. For concentric spheres, analytic solutions for the conductive regime were reported by Makc \& Hardee [3]. The dependence of the Nusselt number $N u$ on the Rayleigh number $R a$ on the transition from the conductive to the convective steady solutions was obtained by Teertstra et al. 4. The stability analysis of the convective regime has deserved wide attention, see for example 5 . Numerical simulations of the concentric configuration can be found for a variety of numerical methods, in particular for the study of the transition to turbulence [6, 7, 8, 9, 10,

Little is known of the inverted configuration. The case of an outer shell hotter than an inner concentric shell was experimentally and numerically studied by Futterer et al. using silicone oil [11. They found unsteady periodic flows consisting of cold blobs dripping from the inner sphere at a frequency that became irregular through a period-doubling process as $R a$ increases.

While concentric configurations have received much attention, few studies can be found about the eccentric ones. In the present work, we report a numerical exploration of the natural convection between eccentric spherical shells at fixed temperatures (see figure 1). Simulations were performed using a three dimensional, two-component lattice Boltzmann method (LBM) that approximates solutions to the Oberbeck-Boussinesq equations of thermal convection [12. This method has been used successfully for the simulation of natural convection phenomena [13, 14.

In the next section we present the numerical method and its validation. We compare predicted results with concentric and vertically eccentric numerical and experimental observations found in the literature. We present numerical solutions for different eccentric configurations in the third section. The transition from conductive to convective heat transport is characterised, and a series of steady and unsteady patterns and behaviour are presented in this section. In the last section we summarise our observations and motivate future work.

\footnotetext{
*Author to whom correspondence should be addressed: cmi.ciencias@ciencias.unam.mx

†Physics Department, School of Science, Universidad Nacional Autónoma de México
} 


\section{The thermal LBM}

To simulate natural convection phenomena we used a D3Q19 two-component lattice Boltzmann equation. The method is a direct three dimensional extension of that proposed by Innamuro in 2002 [12]. Space is discretized using a cubic lattice where a density $f_{k}$ and a temperature $\mathrm{g}_{k}$ distribution functions are computed. The distribution functions are then used to compute the fluid velocity $\boldsymbol{u}$ and temperature $T$ at the lattice nodes. Lattice spacing as well as time steps can be conveniently set to unity. At every node $\boldsymbol{r}$ in the lattice, the distribution functions evolve in time according to

$$
\begin{aligned}
& f_{k}\left(\boldsymbol{r}+\boldsymbol{e}_{k}, t+1\right)=f_{k}(\boldsymbol{r}, t)-\frac{1}{\tau}\left[f_{k}(\boldsymbol{r}, t)-f_{k}^{e q}(\boldsymbol{r}, t)\right]+G_{k}, \\
& g_{k}\left(\boldsymbol{r}+\boldsymbol{e}_{k}, t+1\right)=g_{k}(\boldsymbol{r}, t)-\frac{1}{\tau_{g}}\left[g_{k}(\boldsymbol{r}, t)-g_{k}^{e q}(\boldsymbol{r}, t)\right] .
\end{aligned}
$$

The coefficients $\tau$ and $\tau_{g}$ represent relaxation times and are related to the fluid kinematic viscosity $\nu=(\tau-1 / 2) / 3$ and thermal diffusivity $\alpha=\left(\tau_{g}-1 / 2\right) / 3$. The local equilibrium distribution functions $f_{k}^{e q}$ and $g_{k}^{e q}$ are given by

$$
\begin{aligned}
f_{k}^{e q} & =\rho w_{k}\left[1+3 \boldsymbol{e}_{k} \cdot \boldsymbol{u}+\frac{9}{2}\left(\boldsymbol{e}_{k} \cdot \boldsymbol{u}\right)^{2}-\frac{3}{2} u^{2}\right], \\
g_{k}^{e q} & =T w_{k}\left[1+3 \boldsymbol{e}_{k} \cdot \boldsymbol{u}\right] .
\end{aligned}
$$

The equilibrium distributions depend on the macroscopic fields $\boldsymbol{u}, T$ and $\rho$, the mass density, and must be computed every time step through

$$
\begin{aligned}
\rho(\boldsymbol{r}, t) & =\sum_{k=0}^{18} f_{k}(\boldsymbol{r}, t), \\
\rho \boldsymbol{u}(\boldsymbol{r}, t) & =\sum_{k=0}^{18} \boldsymbol{e}_{k} f_{k}(\boldsymbol{r}, t), \\
T(\boldsymbol{r}, t) & =\sum_{k=0}^{18} g_{k}(\boldsymbol{r}, t)
\end{aligned}
$$

The constants $w_{k}$ in the equilibrium functions definitions (3) and (4) take the values $w_{0}=1 / 3, w_{k}=1 / 18$ for $k=1, \ldots, 6$ and $w_{k}=1 / 36$ for $k=7, \ldots, 18$. The last term in (1) is related to the body force and gives the buoyancy term in the Boussinesq equations. It is defined as $G_{k}=-3 \beta w_{k}\left(T(\boldsymbol{r}, t)-T_{0}\right) \boldsymbol{e}_{k} \cdot \boldsymbol{g}$, where $\beta$ is the coefficient of thermal expansion of the fluid, $\boldsymbol{g}$ the acceleration due to gravity and $T_{0}$ a reference temperature taken as the average of the temperatures of the inner and the outer shells.

The set of microscopic velocities $\left\{\boldsymbol{e}_{k}: k=0, \ldots, 18\right\}$ is given by

$$
\begin{array}{r}
\boldsymbol{e}_{0}=(0,0,0), \quad \boldsymbol{e}_{1}=-\boldsymbol{e}_{4}=(1,0,0), \quad \boldsymbol{e}_{2}=-\boldsymbol{e}_{5}=(0,1,0), \quad \boldsymbol{e}_{3}=-\boldsymbol{e}_{6}=(0,0,1), \\
\boldsymbol{e}_{7}=-\boldsymbol{e}_{10}=(1,1,0), \quad \boldsymbol{e}_{8}=-\boldsymbol{e}_{11}=(1,0,1), \quad \boldsymbol{e}_{9}=-\boldsymbol{e}_{12}=(0,1,1), \\
\boldsymbol{e}_{13}=-\boldsymbol{e}_{16}=(-1,1,0), \quad \boldsymbol{e}_{14}=-\boldsymbol{e}_{17}=(-1,0,1), \quad \boldsymbol{e}_{15}=-\boldsymbol{e}_{18}=(0,-1,1) .
\end{array}
$$

Equations (1) and (2), with the choice microscopic velocities, provide an algorithm for updating all the distribution functions $f_{k}$ and $g_{k}$ at a given node in the lattice, as long as its 18 nearest neighbours in the lattice are inside the fluid domain. For nodes close enough to a solid wall, the distribution functions coming from neighbouring nodes outside the fluid domain must be provided as a boundary condition for the method. We choose to adopt the set of boundary conditions proposed by [14 for curved solid walls at fixed temperatures. In this boundary conditions, distribution functions that should provide the node outside the fluid domain are extrapolated considering that $T$ and $\boldsymbol{u}$ are prescribed at the point in the boundary that intersects the line joining the nodes in question.

The method was implemented to run in parallel in Graphic Processing Units (GPU) because of the large number of nodes needed in the simulation of three dimensional flows. Typically, around $10^{7}$ nodes where used to obtain unsteady flows and simulations took a few days running on a single GPU. 
(a)

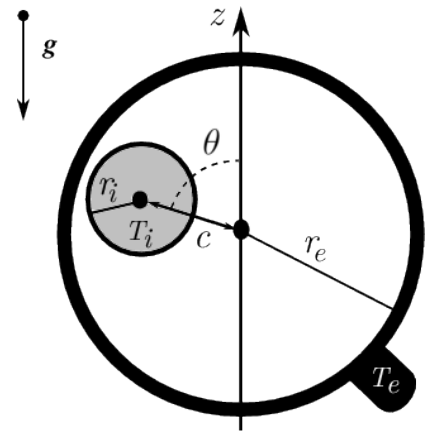

(b)

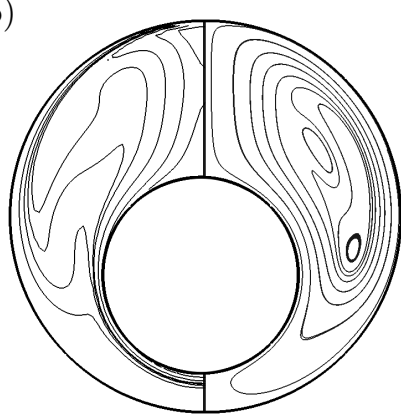

Figure 1: (a) Schematic representation of the problem. (b) Steady numerical solution for $\eta=0.5, \theta=\pi$, $\epsilon=0.6$ and $R a=1.875 \times 10^{5}$. The numerical solutions is symmetric with respect to the vertical axis and the figure shows isothermal curves in the left half of a plane of symmetry and stream lines in the right half.

\section{Problem statement and validation}

A fluid of initial density $\rho_{0}$ fills the gap between two eccentric spherical shells at fixed positions. The inner sphere of radius $r_{i}$ is at temperature $T_{i}$ and the external sphere of radius $r_{e}$ is at a higher temperature $T_{e}$. The position of the centre of the inner sphere is given by the polar angle $\theta$ and the distance to the external sphere centre $c$, as shown in figure 1(a). The vertical plain containing the centres of both spheres is a plane of symmetry of the fluid domain.

The thermal LBM presented in the previous section approximates solutions to the Boussinesq equations describing the convective flow of the stated problem [12]. Scaling lengths with $L=r_{e}-r_{i}$, temperatures with $\Delta T=T_{i}-T_{e}$, velocities with $\alpha / L$ and pressure with $\rho_{0} \alpha^{2} / L^{2}$, the convective flow is defined by the non-dimensional eccentricity $\epsilon$, the polar angle $\theta$, the aspect ratio $\eta$, the Rayleigh number $R a$ and the Prandtl number $\operatorname{Pr}$ given by

$$
\epsilon=\frac{c}{r_{e}}, \quad \eta=\frac{r_{e}}{r_{i}}, \quad R a=\frac{g \beta \Delta T L^{3}}{\nu \alpha}, \quad \operatorname{Pr}=\frac{\nu}{\alpha} .
$$

For all the flows studied $\operatorname{Pr}=0.7$, corresponding to air, and $T_{i}>T_{e}$ which gives $R a>0$.

To validate the code we compared with a variety of results in the literature. Numerical results reproduced the transition from conductive to convective energy transport observed experimentally for concentric spheres by Teertstra et al. [4] through the measurement of the average Nusselt number on the internal shell, defined here as

$$
N u=\frac{L}{4 \pi r_{e} r_{i} \Delta T} \oint-\frac{\partial T}{\partial n} d A,
$$

where $n$ is the normal direction to the spheres.

The values of $N u$ obtained for steady axisymmetric flows are compared to those of Teertstra et al. in figure 2 for different values of $\eta$, showing a good agreement with experiments. The insets in figure 2 (a) show isothermal curves (left half) and stream lines (right half) obtained with the code on a plane of symmetry. Each inset shows the obtained flow patterns and plume formation through three characteristic regions, roughly divided by the vertical dotted lines. These regions correspond to the conductive and convective regimes, and a transitional region in between them.

A linear fit of the numerical results for the steady convective region in the range $0.2<\eta<0.7$ suggests a power law relation given by

$$
N u=0.36(1-\eta) R a^{0.23 \eta+0.14} .
$$

This relation is consistent with correlations obtained by Raithby \& Hollands [15, Scanlan et al. [16] and Feldman \& Colonius [6].

In figure 1(b) isothermal curves and stream lines are shown for a case of vertically eccentric spheres. The flow pattern is in good agreement to that observed experimentally by Powe et al. 2] for the set of parameters in the region they named "Steady Multiple Interior Cells". 
(a)
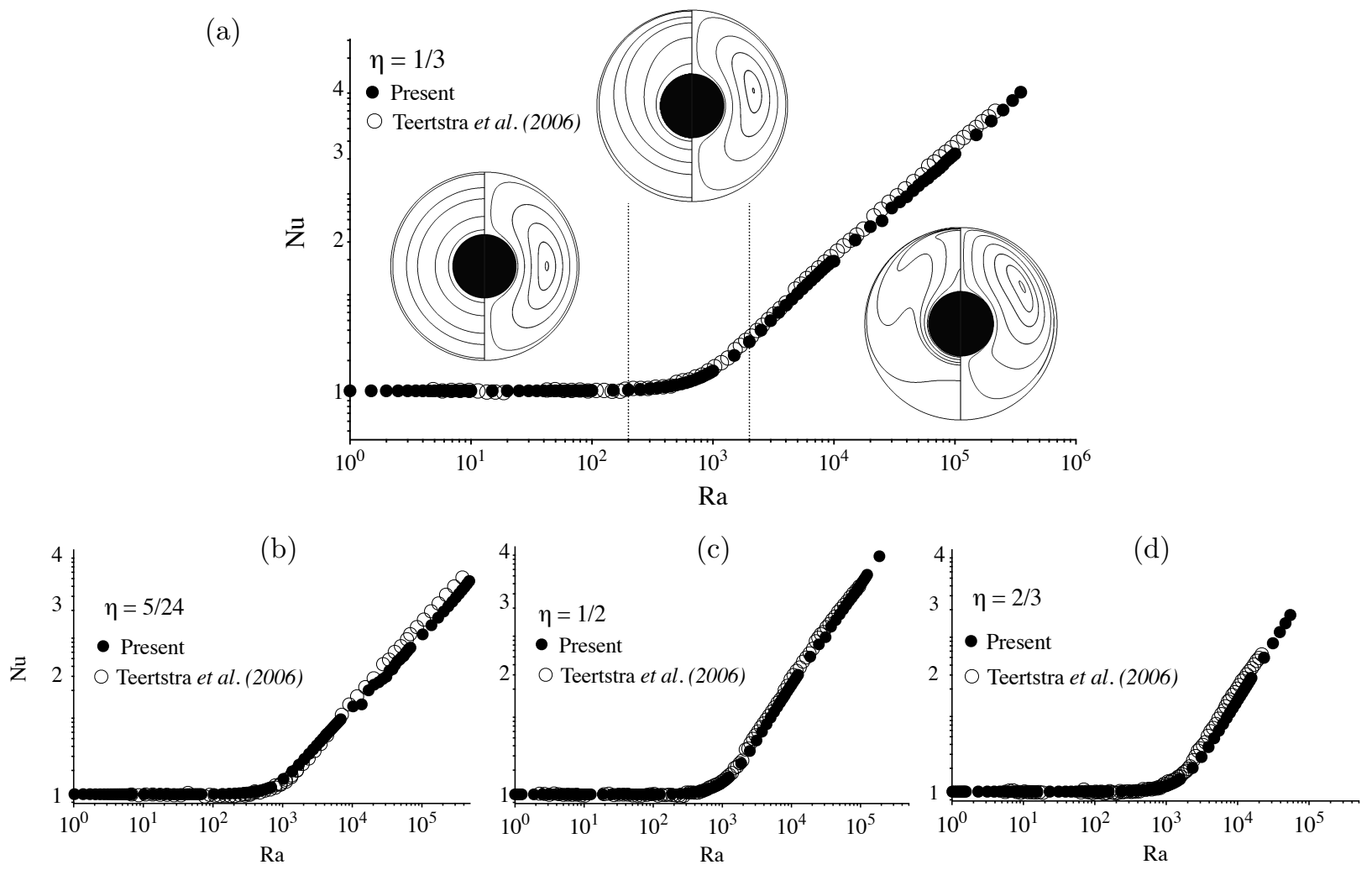

Figure 2: Comparison of the steady state $N u$ numbers obtained with LBM and experiments performed by Teertstra et al. [4] for concentric shell and different aspect ratios. $\eta=1 / 3$ in (a), $\eta=5 / 24$ in (b), $\eta=1 / 2$ in (c) and $\eta=2 / 3$ in (d). Insets in (a) show typical isothermal lines in the left half, and steam lines in the right half, found in the three regions divided by the dotted vertical lines.

Convection between concentric spheres for $\eta=0.714$ served to validate the code with unsteady three dimensional flows. Figure 3 shows numerical solutions for $R a=4.03 \times 10^{4}$, just above the critical value $\left(R a=3.96 \times 10^{4}\right)$ predicted by the linear stability analysis presented by Travnikov et al. [5]. The nondimensional temperature $\Theta=\left(T-T_{i}\right) / \Delta T$ and azimuthal velocity component $u_{\phi}$ on the midrange spherical surface $r=\left(r_{e}+r_{i}\right) / 2$ show an azimuthal mode $m=10$, which is also the critical wave number predicted by linear stability. This unsteady periodical flow was obtained by letting the solution reach a steady state at $R a=3.8 \times 10^{4}$ before increasing its value to $R a=4.03 \times 10^{4}$. The pattern of $u_{\phi}$ is very similar to that obtained by Scurtu et al. 7. for travelling and pulsating waves of mode $m=10$ using spectral methods, and those obtained by Feldman \& Colonius [6] for mode $m=12$ at higher $R a$ values using openFoam algorithms. When $R a$ was increased to $5 \times 10^{4}$, the solution showed an $m=11$ mode over many oscillations before changing to an $m=12$ mode, which is consistent with results in [7, 6]. The non-dimensional velocity $u_{\phi}$ shown in figure 3 rescaled taking $\alpha=2 \times 10^{-5} \mathrm{~m}^{2} / \mathrm{s}$ and $L=0.1 \mathrm{~m}$, predicts azimuthal velocities of the order of $0.1 \mathrm{~m} / \mathrm{s}$.

In general, steady and time averaged $N u$ numbers predicted by the code are in good agreement with the corresponding values previously reported for steady and periodic flows. This is summarised in Table 1 . where numbers with an asterisk superscript denote a time average $N u$ of a periodic solution. Notice that a steady state was obtained for $\eta=0.667$ and $R a=10^{4}$, this is in agreement with experiments in [4] (see figure 2(c)) .

Typical simulations required $4 \times 10^{5}$ time steps and the three dimensional fluid domain was represented by as much as $2 \times 10^{7}$ grid points for unsteady solutions. Using a Tesla C2075 parallel processor, runs took 
(a)

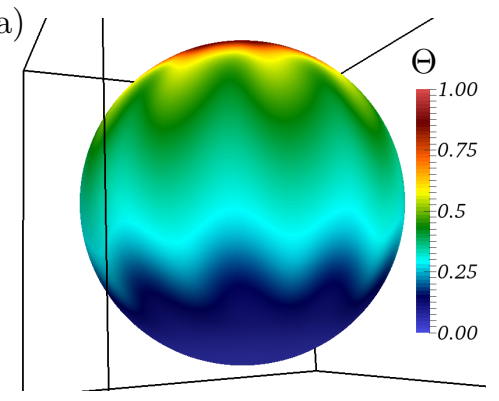

(c)

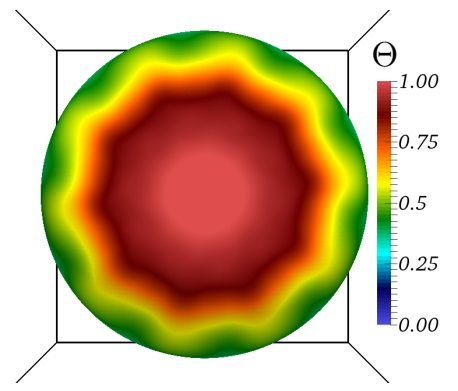

(b)

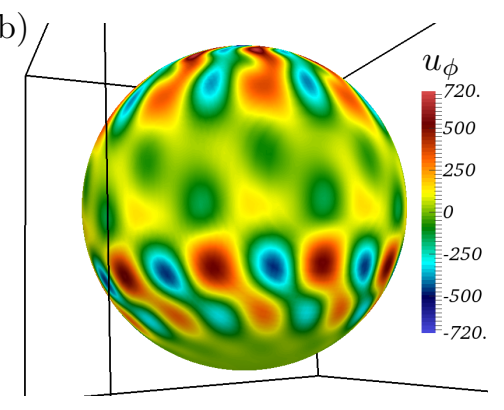

(d)

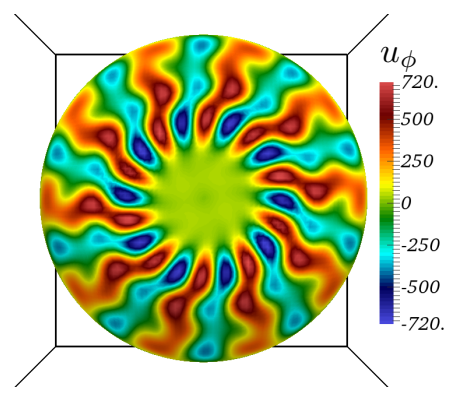

Figure 3: Numerical solutions of the non-dimensional temperature and azimuthal velocity component for $\eta=0.714, \epsilon=0$ and $R a=4.03 \times 10^{4}$ on the midrange spherical surface $r=\left(r_{e}+r_{i}\right) / 2$ showing an azimuthal mode $m=10$. In (a) and (c) $\Theta$ is shown from the top and lateral views respectively. In (b) and (d) $u_{\phi}$ is shown from the top and lateral views respectively.

between three and six days.

\section{Eccentric configurations}

For the case of concentric shells, the average $N u$ number defined in 100 represents the average heat flux on either the external or the internal sphere, divided by $k \Delta T \eta / L$ or $k \Delta T /(L \eta)$ respectively. The latter expressions are the average heat flux for the convection problem in concentric spheres in the external and internal spheres respectively. Defined in this way $N u$ tends to one in both spheres for small values of $R a$ as heat transport is dominated by conduction (see figure 2). For eccentric spheres we defined the average $N u$ number accordingly as

$$
N u=\frac{L}{4 \pi r^{2} \Delta T N u^{*}} \oint-\frac{\partial T}{\partial r} d A,
$$

Table 1: Steady and time averaged $N u$ numbers

\begin{tabular}{ccccccc|c}
\hline \hline$\eta$ & Ra & Present & Ref. [6] & Ref. [8] & Ref. [9] & Ref. [10] & Eq. [1] \\
\hline \hline 0.5 & $10^{2}$ & 1.0077 & 1.0217 & 1.001 & 1.000 & & \\
0.5 & $10^{3}$ & 1.0865 & 1.104 & 1.0990 & 1.1310 & 1.1021 & 1.0478 \\
0.5 & $10^{4}$ & 1.8977 & 1.9665 & 1.9730 & 1.9495 & 1.9110 & 1.88848 \\
0.5 & $10^{5}$ & 3.3077 & 3.4012 & 3.4890 & 3.4648 & 3.355 & 3.3906 \\
\hline 0.667 & $10^{3}$ & 1.0415 & 1.04825 & 1.001 & 1.00115 & & \\
0.667 & $10^{4}$ & 1.7295 & $1.793^{*}$ & 1.073 & 1.07138 & & 1.7886 \\
\hline 0.833 & $10^{3}$ & 1.0118 & 1.011 & 1.0 & 1.0018 & & \\
0.833 & $10^{4}$ & $1.5993^{*}$ & $1.6523^{*}$ & 1.001 & 1.0028 & & \\
\hline
\end{tabular}




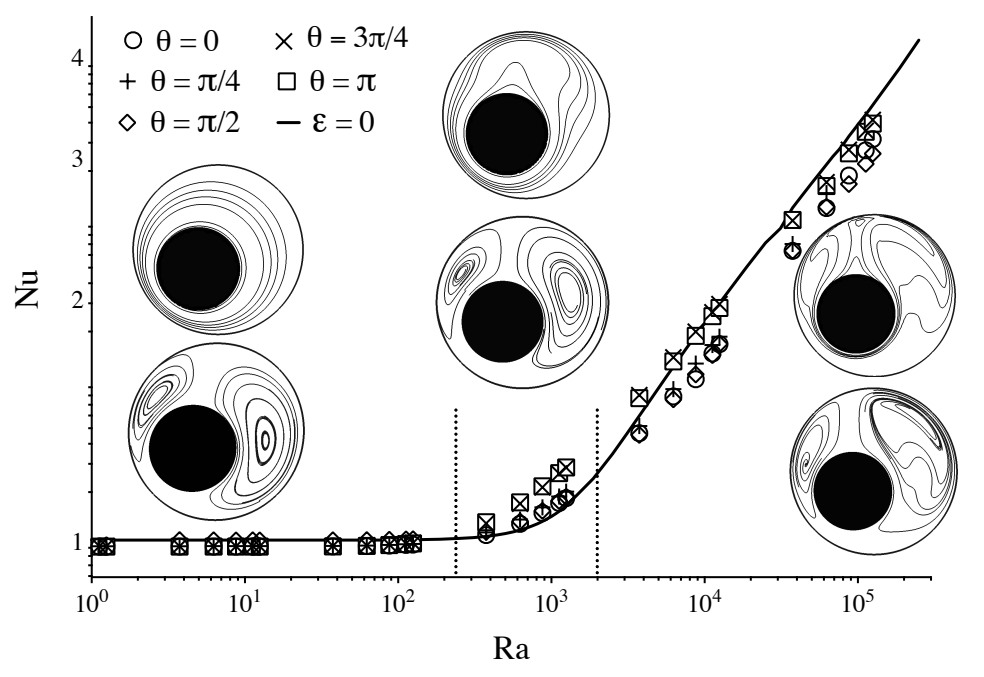

Figure 4: Numerical prediction of the steady $N u$ number showing the convective to conductive transition for eccentric configurations for $\eta=0.5$ and $\epsilon=0.6$. Solutions at polar angles $\theta=0, \pi / 4, \pi / 2,3 \pi / 4, \pi$ are compared with the concentric solution represented by the continuous line. The insets show the typical isothermal curves (top) and stream lines (bottom) for $\theta=3 \pi / 4$ on the plane of symmetry in the conductive and convective regions, and a transitional region in between them marked by the vertical dotted lines.

where $r$ takes the values $r_{e}$ and $r_{i}$, and $N u^{*}$ is the average Nusselt number for the heat conduction problem between eccentric spheres obtained by Alassar [17. Through bispherical coordinates, $N u^{*}$ is expressed as an infinite series

$$
N u^{*}=\frac{2 a L}{r^{2} \Delta T} \sum_{n=0}^{\infty} \frac{1}{e^{(2 n+1) \xi_{e}}-e^{(2 n+1) \xi_{i}}},
$$

where $r$ takes the values $r_{e}$ and $r_{i}, \xi_{e}=\sinh ^{-1}\left(a / r_{e}\right), \xi_{i}=\sinh ^{-1}\left(a / r_{i}\right)$ and

$$
a=\sqrt{\left(c+r_{i}+r_{e}\right)\left(c+r_{i}-r_{e}\right)\left(c-r_{i}+r_{e}\right)\left(c-r_{i}-r_{e}\right)} / 2 c
$$

determines the foci of the bispherical coordinates.

We first searched for steady solutions for fixed values of $\eta$ and $\epsilon$ and different angles $\theta$. In figure 4 appears the behaviour of the steady $N u$ number as $R a$ increases for $\eta=0.5, \epsilon=0.6$, and the set of angles $\theta=0, \pi / 4, \pi / 2,3 \pi / 4, \pi$. Numerical solutions show a similar behaviour of that observed in concentric spheres, given by the continuous line in the figure. There is a transition from a conductive to a convective regime at roughly the same values of the $R a$ number as the concentric solutions. At angles $\theta=3 \pi / 4$ and $\pi$ the steady convective regime seems to be reached at lower values of $R a$ than the rest of cases shown in the figure. Also, the slopes of the convective regime are slightly lower for the eccentric configurations. A linear fit suggest exponents of values around 0.245 for $\theta>\pi / 2,0.225$ for $\theta<\pi / 2$ and its lowest value of 0.2 for $\theta=\pi / 2$. Insets show the evolution of isothermal curves and steam lines. On the other hand, eccentric simulations become unstable at lower values of the $R a$ number when compared to concentric ones.

Beyond the steady convective region a series of periodic solutions where found. Starting from an oscillating $N u$ number at the inner and outer spheres with a clear frequency, as the $R a$ number increased unsteady flow behaviour became irregular through what seamed as a period doubling process. The behaviour $N u$ along non-dimensional time $t$ is shown in figure 5 for the case of $\eta=0.5, \epsilon=0.8$ and $\theta=\pi / 4$. Taking $\alpha=2 \times 10^{-5} \mathrm{~m}^{2} / \mathrm{s}$ and $L=0.1 \mathrm{~m}$, the times on figure 5 must be rescaled with a time of $500 \mathrm{~s}$ and so the figure would show a lapse of $10 \mathrm{~s}$. 


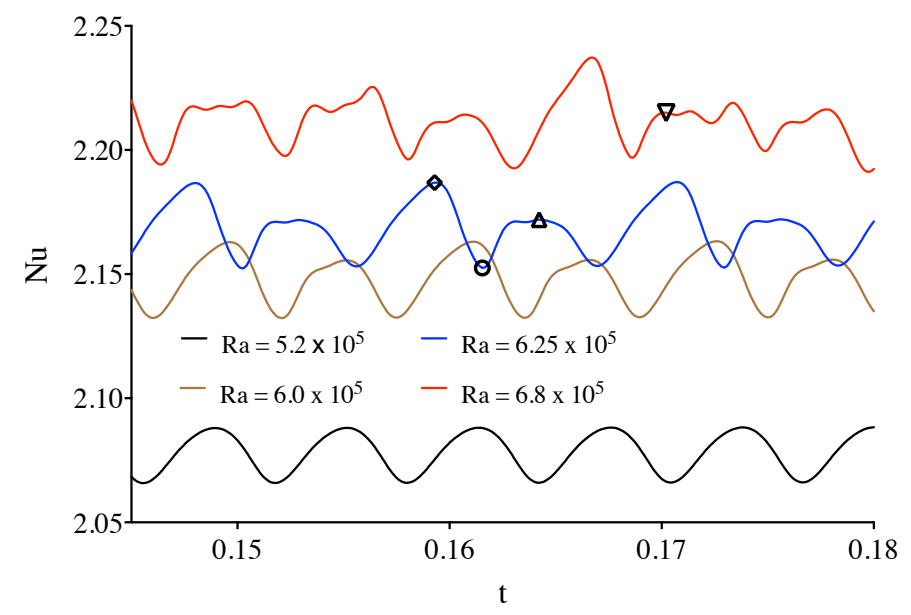

Figure 5: Time evolution of the average $N u$ number calculated on the external sphere for the case of $\eta=0.5$, $\epsilon=0.8$ and $\theta=\pi / 4$ and increasing values of the $R a$ number. The different curves show a periodic behaviour of increasing complexity as the $R a$ number grows. Details of the flows at the points indicated with the symbols $\diamond, \circ, \triangle$ and $\nabla$ are shown in figures 6 and 7

Figure 6 shows in columns some details of the unsteady convecive flow for $R a=6.25 \times 10^{5}$ at three times, indicated in figure 5, when the $N u$ number reaches an absolute maximum value, the following minimum and the next local maximum respectively (the symbols $\diamond, \circ$ and $\Delta$ in figure 5). The top row shows the isothermal surface $\Theta=0.7$, the row in the middle shows the temperature field on the plane of symmetry of the flow domain and the bottom row shows a set of stream lines superposed to the surface of $\Theta=0.7$.

Observations suggest that isothermal surfaces suffer an instability in the direction transversal to the plane of symmetry, generating a sort of "tail" at the top of the surface that seem to flap vertically. This oscillation is accompanied by currents coming from the "front" of the inner sphere when the $N u$ number reaches its peaks (see figures 6(g) and 6(i)). Lower values of $N u$ appear with smoother convection cells and flow patterns within the flow (see figure 6(h)). At higher $R a$ numbers, the currents coming from the front start to oscillate horizontally showing a new instability of the isothermal surfaces. This can be seen in figure 7. corresponding to the point marked with the symbol $\nabla$ in the curve of $R a=6.8 \times 10^{5}$ in figure 5 .

\section{Conclusions}

It is remarkable that such a simple numerical model as LBM can reproduce steady and unsteady three dimensional natural convection flows. Additionally, the simplicity of the algorithm allows for an efficient implementation on massively parallel architectures. This was the motivation for exploring natural convection in eccentric annuli configurations with this method. Although there is much work done in concentric configurations, eccentric ones have received little attention and our observations contribute to the complete picture of natural convection in spherical annuli.

When validated with numerical and experimental results of the concentric configuration, results suggested a power law correlation of the average $N u$ number on the spherical boundaries with the $R a$ number and the aspect ratio, see equation (11), close to what has been proposed in previous work (see [15, 16, 6]). When simulating eccentric configurations, result suggested a transition from a conductive regime to a steady convective regime similar to that found in the concentric case. Convective regimes are reached at lower $R a$ numbers and $N u$ grows at a smaller rate in eccentric configurations. The lack of symmetry should produce more mixing in and lower the heat flux observed in the convective regime. 
(a)

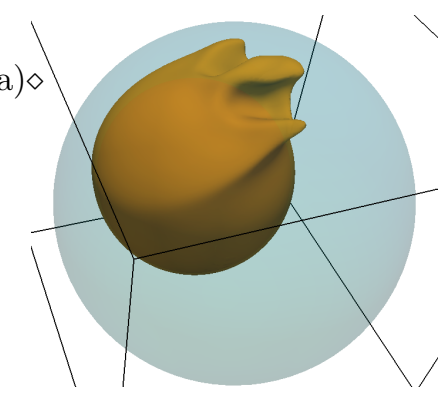

(d) $\diamond$
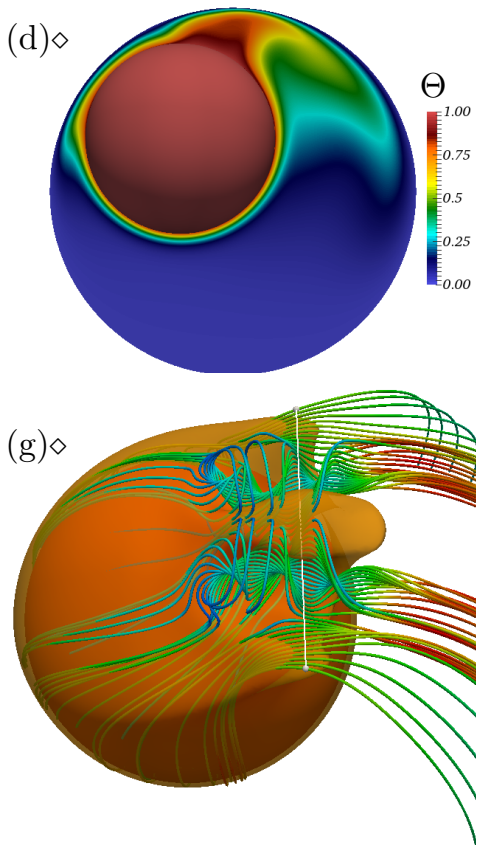

(b)

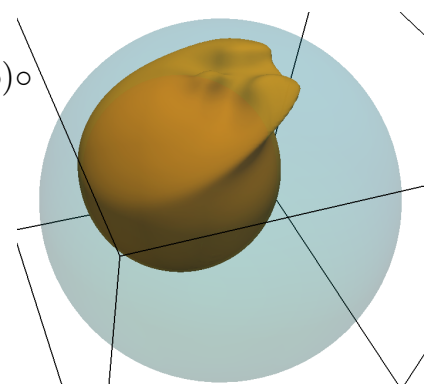

(e)o

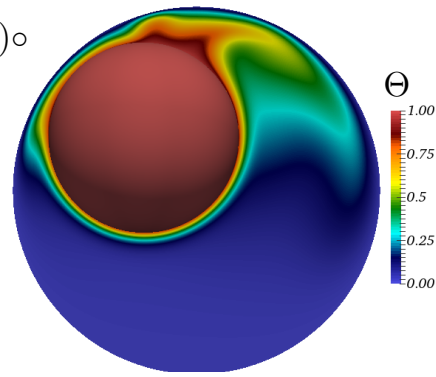

(h)。

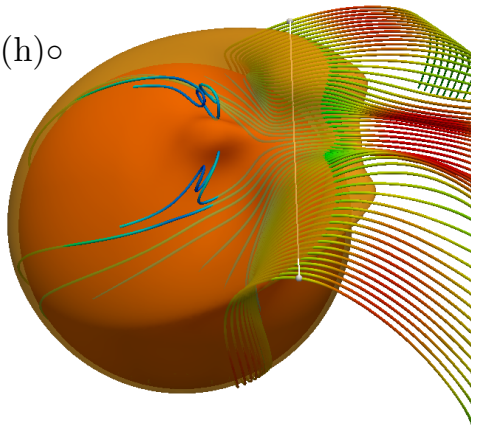

(c) $\triangle$

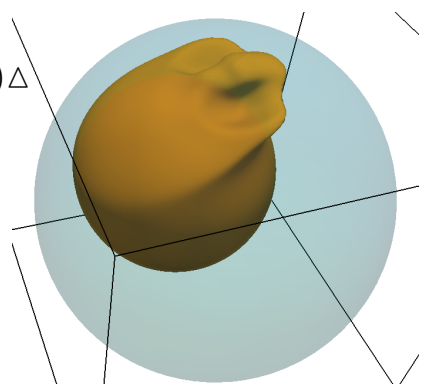

(f) $\triangle$

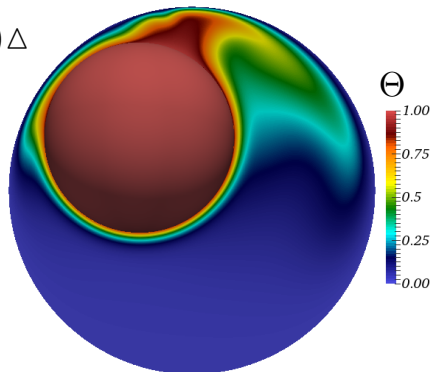

(i) $\triangle$

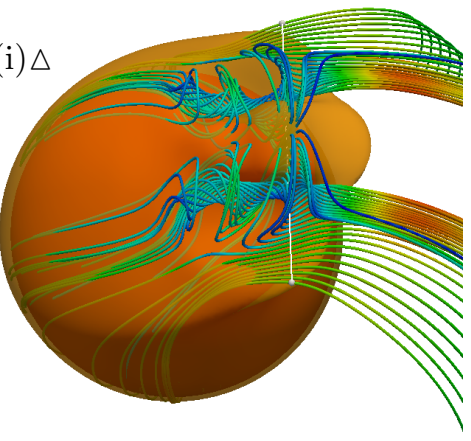

Figure 6: Results for the case of $\eta=0.5, \epsilon=0.8, \theta=\pi / 4$ and $R a=6.8 \times 10^{5}$ at the times indicated by the points $\diamond, \circ$ and $\Delta$ in figure 5 . The row at the top show surface $\Theta=0.7$ located inside the enclosing sphere. The row in the middle shows a slice of the temperature field at the plane of symmetry of the configuration. The bottom row shows stream lines seeded on the white line coloured with the magnitude of the velocity.

Eccentric convective states also become unstable at lower values of $R a$. As $R a$ grows, flow structure and the $N u$ number present periodic fluctuations in time. $N u$ oscillations are related to the deformation of isothermal surfaces. These show a modulation transversal to the vertical plane of symmetry and, as $R a$ grows, surfaces start to oscillate in the horizontal direction as well. Similar behaviour of the periodicity of the flow and heat flux was found in natural convection in a concentric annuli for an inner sphere colder than the outer one [1].

Results here presented are encouraging and suggest that many different examples of three dimensional natural convection phenomena between a body and its enclosure can be studied using LBM. Specially given the simplicity to impose boundary conditions on curved surfaces in LBM, and the efficiency that exhibits under fine grain parallelism.

\section{Acknowledgements.}

We are grateful to Dr. P. M. Teertstra for providing experimental data. We are also thankful to Dr. R. Rechtman for his fruitful suggestions. 


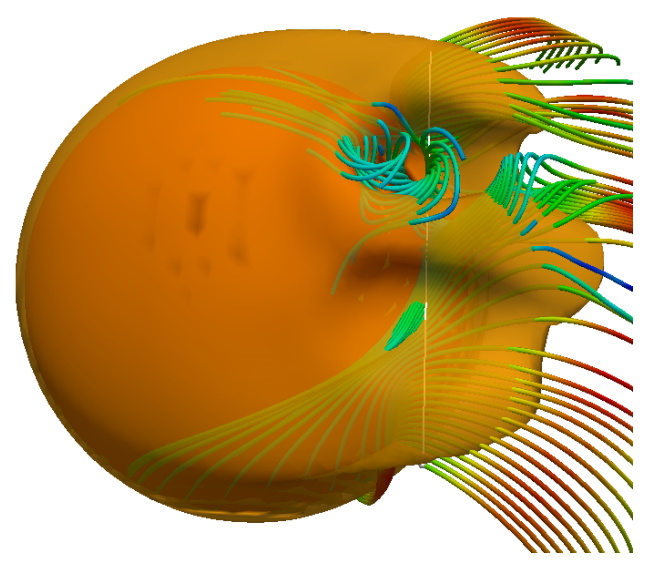

Figure 7: Isothermal surface $\Theta=0.7$ superimposed to a set of stream lines seeded on the white line at the time indicated by $\nabla$ in figure 5 . Stream lines are coloured with the magnitude of the velocity. Parameter values are $\eta=0.5, \epsilon=0.8, \theta=\pi / 4$ and $R a=6.8 \times 10^{5}$.

\section{References}

[1] E. Bishop, L. Mack, J. Scanlan. Heat transfer by natural convection between concentric spheres. Int. J. Heat Mass Transfer 9 (1966) 649-662.

[2] R. Powe, R. Warrington, J. Scanlan. Natural convective flow between a body and its spherical enclosure. Int. J. Heat Mass Transfer 23 (1980) 1337-1350.

[3] L. R. Mack, H. C. Hardee. Natural convection between concentric spheres at low Rayleigh numbers. Int. J. Heat Mass Transfer 11 (1968) 387-396.

[4] P. Teertstra, M. Yovanovih, J.R. Culham. Natural convection measurements for a concentric spherical enclosure. J. Heat Transfer 128 (2006) 580-587.

[5] V. Travnikov, K. Eckert, S. Odenbach. Linear stability analysis of the convective flow in a spherical gap with $\eta=0.714$. Int. J. Heat Mass Transfer 80 (2015) 266-273.

[6] Y. Feldman, T. Colonius. On a transitional and turbulent natural convection in spherical shells. Int. J. Heat Mass Transfer 64 (2013) 514-525.

[7] N. Scurtu, B. Futterer, C. Egbers. Pulsating and travelling wave modes of natural convection in spherical shells. Physics of Fluids 22 (2010) 114108.

[8] H. Chu, T. Lee. Transient natural convection heat transfer between concentric spheres. Int. J. Heat Mass Transfer 36 (1993) 3159-3170.

[9] A. Dehghan, K. Masih. Numerical simulation of buoyancy-induced turbulent flow between two concentric isothermal spheres. Heat Transfer Eng. 31 (2010) 33 ?44.

[10] C. Chiu, W. Chen. Transient natural convection heat transfer between concentric and vertically eccentric spheres. Int. J. Heat Mass Transfer 39 (1996) 1439?1452.

[11] B. Flutterer, A. Bruckcs, R. Hollerbach, C. Egbers. Thermal blob convection in spherical shells. Int. J. Heat Mass Transfer 50 (2007) 4079-4088. 
[12] T. Inamuro, M. M. Yoshino, H. Inoue, R. Mizuno, F. Ogino. A lattice Boltzmann method for a binary miscible fluid mixture and its application to a heat-transfer problem. J. Comput. Phys. 179 (2002) 201215.

[13] F. Mandujano, R. Rechtman. Thermal levitation. J. Fluid Mech. 606 (2008) 105-114.

[14] A. A. Mehrizi, M. Farhadi, H. H. Afrouzi, S. Shayamehr, H. Lotfizadeh. Lattice Bolttzmann simulation of natural convection flow around a horizontal cylinder located beneath an insulation plate. J. Theor. Appl. Mech. 51 (2013) 729-739.

[15] G. Raithby, K. Hollands. A general method of obtaining approximate solutions to laminar and turbulent free convection problems. Adv. Heat Transfer 11 (1975) 265-315.

[16] J. Scanlan, E. Bishop, R. Powe. Natural convection heat transfer between concentric spheres. Int. J. Heat Mass Transfer 13 (1970) 1857-1872.

[17] R. S. Alassar. Conduction in eccentric spherical annuli. Int. J. Heat Mass Transfer 54 (2011) 3796-3800. 